\title{
Chironomidae Types in the Reference Collection of the Laboratory of Ecology of Aquatic Insects, São Carlos, Brazil
}

\author{
Fabio Laurindo da Silva ${ }^{1 *}$, Susana Trivinho-Strixino ${ }^{2}$ and Caroline Silva Neubern de Oliveira ${ }^{2}$ \\ ${ }^{1}$ Museum of Comparative Zoology, Department of Organismic and Evolutionary Biology, Harvard Uni- \\ versity, 26 Oxford Street, Cambridge, MA 02138, USA. \\ E-mail: laurindodasilva@fas.harvard.edu \\ ${ }^{2}$ Laboratory of Ecology of Aquatic Insects, Department of Hydrobiology, Federal University of São Car- \\ los, P.O. Box 676, 13565-905, São Carlos, SP, Brazil. \\ E-mails: strixino@ufscar.br, csneubern@gmail.com
}

*Corresponding author.

\begin{abstract}
The Chironomidae (Insecta: Diptera) type collection at the Laboratory of Ecology of Aquatic Insects (LEIA - UFSCar) is reviewed. It comprises 103 primary types, as well as 95 paratypes, mostly resulting from research by $\mathrm{S}$. Trivinho-Strixino and G. Strixino. Notes updating the taxonomic status are provided for some species.

\section{Introduction}

The type collection of Chironomidae at the Laboratory of Ecology of Aquatic Insects (LEIA - UFSCar) comprises numerous slide mounts, as well as large amounts of adult and immature specimens preserved in $70 \%$ ethanol. It is an important chironomid collection, especially regarding the Neotropical region. Moreover, it possesses valuable yet unstudied material mainly from south-east Brazil. It is historically relevant, but not well known. The largest part of this material results from research activities of S. Trivinho-Strixino and G. Strixino, who during more than three decades at the LEIA UFSCar described numerous species.
\end{abstract}

The annotated checklist presented below (Table 1) was generated during a research visit of the present first author to LEIA - UFSCar in March 2015, which was partly financed by a Putnam expedition grant from the Museum of Comparative Zoology at Harvard University.

\section{Results}

The collection at the LEIA - UFSCar includes holotypes for 103 species of Chironomidae, as well as 95 paratypes, all slide mounted, with a total of 109 species represented. Most of the types belong to the subfamilies Chironominae and Tanypodinae, but there are also types of Orthocladiinae, Podonominae and Telmatogetoninae. In the annotated checklist presented below (Table 1), all species names and references were checked with the corresponding literature. The notes on the species' taxonomic status are based on Systema Dipterorum (Pape and Thompson 2013), as well as on published papers, keys (e.g. Cranston and Epler 2013, Spies and Reiss 1996, Trivinho-Strixino and Sanseverino 2003, Sanseverino et al. 2010) and/or on information from collection labels.

Table 1. Type specimens of Chironomidae deposited in the collection of the Laboratory of Ecology of Aquatic Insects (LEIA - UFSCar). Data in the "Type" and "Sex/Stage" columns refer to what is housed at UFSCar only, and that for some species this is less than the full material listed in the respective original publication. Abbreviations: $\mathrm{H}=$ holotype, $\mathrm{P}=$ paratype, $\mathrm{f}=$ female, $\mathrm{l}=$ larva, $\mathrm{m}=$ male, $\mathrm{p}=$ pupa.

$\begin{array}{lllll}\begin{array}{l}\text { Type } \\ \text { number }\end{array} & \text { Original genus } & \text { Original species } & \begin{array}{l}\text { Author/ } \\ \text { Reference }\end{array} & \text { Type } \begin{array}{l}\text { Sex/ } \\ \text { Stage }\end{array}\end{array}$

Chironominae

\begin{tabular}{|c|c|c|c|c|c|}
\hline A1/01-07 & Aedokritus & coffeatus & $\begin{array}{l}\text { Trivinho-Strixino, } \\
\text { 1997: } 13\end{array}$ & $\mathrm{H}, \mathrm{P}$ & $\mathrm{m}, \mathrm{f}, \mathrm{p}, \mathrm{l}$ \\
\hline M2/01-09 & Beardius & cristhinae & $\begin{array}{l}\text { Trivinho-Strixino and } \\
\text { Siqueira, 2007: } 281\end{array}$ & $\mathrm{H}, \mathrm{P}$ & $\mathrm{m}, \mathrm{f}, \mathrm{p}$ \\
\hline M2/37-45 & Beardius & phytophilus & $\begin{array}{l}\text { Trivinho-Strixino and } \\
\text { Strixino, 2000b: } 245\end{array}$ & $\mathrm{H}, \mathrm{P}$ & $\mathrm{m}, 1$ \\
\hline M2/27-33 & Beardius & roquei & $\begin{array}{l}\text { Trivinho-Strixino and } \\
\text { Siqueira, 2007: } 282\end{array}$ & $\mathrm{H}, \mathrm{P}$ & $\mathrm{m}, \mathrm{p}$ \\
\hline
\end{tabular}




\begin{tabular}{|c|c|c|c|c|c|c|}
\hline $\begin{array}{l}\text { Type } \\
\text { number }\end{array}$ & Original genus & Original species & $\begin{array}{l}\text { Author/ } \\
\text { Reference }\end{array}$ & Type & $\begin{array}{l}\text { Sex/Life } \\
\text { stage }\end{array}$ & Note \\
\hline M2/19-24 & Beardius & xylophilus & $\begin{array}{l}\text { Trivinho-Strixino and } \\
\text { Strixino, 2000b: } 246\end{array}$ & $\mathrm{H}, \mathrm{P}$ & $\mathrm{m}, 1$ & \\
\hline $\mathrm{C} 1 / 01$ & Caladomyia & angelae & $\begin{array}{l}\text { Trivinho-Strixino, } \\
\text { 2012: } 11\end{array}$ & $\mathrm{H}$ & $\mathrm{m}$ & \\
\hline $\begin{array}{l}\text { C2/01, } \\
\text { C2/03, } \\
\text { C2/06, } \\
\text { C2/08 }\end{array}$ & Caladomyia & bruneola & $\begin{array}{l}\text { Trivinho-Strixino, } \\
\text { 2012: } 13\end{array}$ & $\mathrm{H}, \mathrm{P}$ & $\mathrm{m}, \mathrm{f}, \mathrm{p}, \mathrm{l}$ & \\
\hline $\mathrm{C} 1 / 02-03$ & Caladomyia & canine & $\begin{array}{l}\text { Trivinho-Strixino, } \\
\text { 2012: } 16\end{array}$ & $\mathrm{H}, \mathrm{P}$ & $\mathrm{m}, \mathrm{p}$ & \\
\hline $\begin{array}{l}\text { C1/06-07, } \\
\text { C1/09-11 }\end{array}$ & Caladomyia & capaopreto & $\begin{array}{l}\text { Trivinho-Strixino, } \\
\text { 2012: } 18\end{array}$ & $\mathrm{H}, \mathrm{P}$ & $\mathrm{m}, \mathrm{p}, 1$ & \\
\hline C1/04-05 & Caladomyia & carolae & $\begin{array}{l}\text { Trivinho-Strixino, } \\
\text { 2012: } 21\end{array}$ & $\mathrm{H}, \mathrm{P}$ & $\mathrm{m}$ & \\
\hline C2/12-19 & Caladomyia & carolensis & $\begin{array}{l}\text { Trivinho-Strixino, } \\
\text { 2012: } 22\end{array}$ & $\mathrm{H}, \mathrm{P}$ & $\mathrm{m}, \mathrm{f}, \mathrm{p}, \mathrm{l}$ & \\
\hline C1/45-48 & Caladomyia & comunis & $\begin{array}{l}\text { Trivinho-Strixino, } \\
\text { 2012: } 26\end{array}$ & $\mathrm{H}, \mathrm{P}$ & $\mathrm{m}, \mathrm{p}, 1$ & \\
\hline $\mathrm{C} 1 / 13$ & Caladomyia & curumim & $\begin{array}{l}\text { Trivinho-Strixino, } \\
\text { 2012: } 29\end{array}$ & $\mathrm{H}$ & $\mathrm{m}, \mathrm{p}, 1$ & \\
\hline C3/04-15 & Caladomyia & friederi & $\begin{array}{l}\text { Trivinho-Strixino and } \\
\text { Strixino, 2000a: } 168\end{array}$ & $\mathrm{H}, \mathrm{P}$ & $\mathrm{m}, \mathrm{f}, \mathrm{p}, \mathrm{l}$ & \\
\hline C1/15-18 & Caladomyia & jaragua & $\begin{array}{l}\text { Trivinho-Strixino, } \\
\text { 2012: } 31\end{array}$ & $\mathrm{H}$ & $\mathrm{m}, \mathrm{p}, \mathrm{l}$ & \\
\hline $\begin{array}{l}\mathrm{C} 2 / 28-29 \\
\mathrm{C} 2 / 32-33 \\
\mathrm{C} 2 / 35\end{array}$ & Caladomyia & kapilei & $\begin{array}{l}\text { Trivinho-Strixino, } \\
\text { 2012: } 33\end{array}$ & $\mathrm{H}, \mathrm{P}$ & $\mathrm{m}, \mathrm{p}, 1$ & \\
\hline $\mathrm{C} 1 / 20-25$ & Caladomyia & yara & $\begin{array}{l}\text { Trivinho-Strixino, } \\
\text { 2012: } 36\end{array}$ & $\mathrm{H}, \mathrm{P}$ & $\mathrm{m}, \mathrm{f}, \mathrm{p}, \mathrm{l}$ & \\
\hline B1/01-13 & Chironomus & amissum & $\begin{array}{l}\text { Correia, Trivinho- } \\
\text { Strixino and } \\
\text { Michailova, 2013: } 134\end{array}$ & $\mathrm{H}, \mathrm{P}$ & $\mathrm{m}, \mathrm{p}, 1$ & 1 \\
\hline M2/30-32 & Chironomus & antonioi & $\begin{array}{l}\text { Correia and Trivinho- } \\
\text { Strixino, 2007: } 57\end{array}$ & $\mathrm{H}, \mathrm{P}$ & $\mathrm{m}, \mathrm{p}, 1$ & \\
\hline M1/42-44 & Chironomus & detriticola & $\begin{array}{l}\text { Correia and Trivinho- } \\
\text { Strixino, 2007: } 54\end{array}$ & $\mathrm{H}, \mathrm{P}$ & $\mathrm{m}, \mathrm{p}, \mathrm{l}$ & \\
\hline M1/48 & Chironomus & fittkaui & $\begin{array}{l}\text { Correia and Trivinho- } \\
\text { Strixino, 2007: } 65\end{array}$ & $\mathrm{H}$ & $\mathrm{m}, \mathrm{p}, 1$ & \\
\hline $\mathrm{F} 1 / 15-26$ & Chironomus & inquinatus & $\begin{array}{l}\text { Correia, Trivinho- } \\
\text { Strixino and } \\
\text { Michailova 2006: } 58\end{array}$ & $\mathrm{H}, \mathrm{P}$ & $\mathrm{m}, \mathrm{f}, \mathrm{p}, \mathrm{l}$ & \\
\hline B2/11-14 & Chironomus & oliveirai & $\begin{array}{l}\text { Correia and Trivinho- } \\
\text { Strixino, 2007: } 63\end{array}$ & $\mathrm{H}, \mathrm{P}$ & $\mathrm{m}, \mathrm{p}, 1$ & \\
\hline $\mathrm{B} 2 / 20-26$ & Chironomus & phytophilus & $\begin{array}{l}\text { Correia and Trivinho- } \\
\text { Strixino, 2007: } 61\end{array}$ & $\mathrm{H}, \mathrm{P}$ & $\mathrm{m}, \mathrm{p}, 1$ & \\
\hline
\end{tabular}




\begin{tabular}{|c|c|c|c|c|c|c|}
\hline $\begin{array}{l}\text { Type } \\
\text { number }\end{array}$ & Original genus & Original species & $\begin{array}{l}\text { Author/ } \\
\text { Reference }\end{array}$ & Type & $\begin{array}{l}\text { Sex/Life } \\
\text { stage }\end{array}$ & Note \\
\hline F1/01-15 & Chironomus & reissi & $\begin{array}{l}\text { Correia, Trivinho- } \\
\text { Strixino and } \\
\text { Michailova 2005: } 30\end{array}$ & $\mathrm{H}, \mathrm{P}$ & $\mathrm{m}, \mathrm{f}, \mathrm{p}, \mathrm{l}$ & \\
\hline B2/01-06 & Chironomus & sancticaroli & $\begin{array}{l}\text { Trivinho-Strixino and } \\
\text { Strixino, 1981: } 333\end{array}$ & $\mathrm{H}, \mathrm{P}$ & $\mathrm{m}, \mathrm{f}$ & 2 \\
\hline M1/01-04 & Cryptochironomus & brasiliensis & $\begin{array}{l}\text { Silva, Trivinho- } \\
\text { Strixino and Oliveira, } \\
\text { 2010: } 19\end{array}$ & $\mathrm{H}, \mathrm{P}$ & $\mathrm{m}, \mathrm{p}, \mathrm{l}$ & \\
\hline M1/08-12 & Cryptochironomus & mantiqueira & $\begin{array}{l}\text { Silva, Trivinho- } \\
\text { Strixino and Oliveira, } \\
\text { 2010: } 23\end{array}$ & $\mathrm{H}, \mathrm{P}$ & $\mathrm{m}, \mathrm{p}, 1$ & \\
\hline M1/15-21 & Cryptochironomus & reshchikovi & $\begin{array}{l}\text { Silva, Trivinho- } \\
\text { Strixino and Oliveira, } \\
\text { 2010: } 27\end{array}$ & $\mathrm{H}, \mathrm{P}$ & $\mathrm{m}, \mathrm{p}, 1$ & \\
\hline E3/07-11 & Endotribelos & bicolor & $\begin{array}{l}\text { Trivinho-Strixino and } \\
\text { Pepinelli, 2015: } 3\end{array}$ & $\mathrm{H}, \mathrm{P}$ & $\mathrm{m}, \mathrm{f}, \mathrm{p}, \mathrm{l}$ & \\
\hline E1/15-17 & Endotribelos & calophylli & $\begin{array}{l}\text { Roque and Trivinho- } \\
\text { Strixino, 2008: } 193\end{array}$ & $\mathrm{H}, \mathrm{P}$ & $\mathrm{m}, \mathrm{p}, 1$ & \\
\hline $\mathrm{E} 2 / 01-02$ & Endotribelos & euterpe & $\begin{array}{l}\text { Roque and Trivinho- } \\
\text { Strixino, 2008: } 196\end{array}$ & $\mathrm{H}, \mathrm{P}$ & $\mathrm{m}, \mathrm{f}, \mathrm{p}, \mathrm{l}$ & \\
\hline $\mathrm{E} 3 / 01-03$ & Endotribelos & ficus & $\begin{array}{l}\text { Roque and Trivinho- } \\
\text { Strixino, 2008: } 201\end{array}$ & $\mathrm{H}, \mathrm{P}$ & $\mathrm{m}, \mathrm{p}, 1$ & \\
\hline E3/39-43 & Endotribelos & fulvidus & $\begin{array}{l}\text { Trivinho-Strixino and } \\
\text { Pepinelli, 2015: } 11\end{array}$ & $\mathrm{H}, \mathrm{P}$ & $\mathrm{m}, \mathrm{f}, \mathrm{p}, \mathrm{l}$ & \\
\hline $\mathrm{E} 2 / 37-42$ & Endotribelos & jaragua & $\begin{array}{l}\text { Trivinho-Strixino and } \\
\text { Pepinelli, 2015: } 17\end{array}$ & $\mathrm{H}, \mathrm{P}$ & $\mathrm{m}, \mathrm{f}, \mathrm{p}, \mathrm{l}$ & \\
\hline E3-25 & Endotribelos & jiboia & $\begin{array}{l}\text { Trivinho-Strixino and } \\
\text { Pepinelli, 2015: } 24\end{array}$ & $\mathrm{H}$ & $\mathrm{m}, \mathrm{p}, \mathrm{l}$ & \\
\hline $\mathrm{E} 2 / 20-27$ & Endotribelos & semibruneus & $\begin{array}{l}\text { Trivinho-Strixino and } \\
\text { Pepinelli, 2015: } 24\end{array}$ & $\mathrm{H}, \mathrm{P}$ & $\mathrm{m}, \mathrm{f}, \mathrm{p}, \mathrm{l}$ & \\
\hline E3/34-42 & Endotribelos & sublettei & $\begin{array}{l}\text { Trivinho-Strixino and } \\
\text { Pepinelli, 2015: } 29\end{array}$ & $\mathrm{H}, \mathrm{P}$ & $\mathrm{m}, \mathrm{f}, \mathrm{p}, \mathrm{l}$ & \\
\hline E1/01-05 & Endotribelos & talaumae & $\begin{array}{l}\text { Roque and Trivinho- } \\
\text { Strixino, 2008: } 203\end{array}$ & $\mathrm{H}, \mathrm{P}$ & $\mathrm{m}, \mathrm{f}, \mathrm{p}, \mathrm{l}$ & \\
\hline L1/01-09 & Goeldichironomus & luridus & $\begin{array}{l}\text { Trivinho-Strixino and } \\
\text { Strixino, 2005: } 441\end{array}$ & $\mathrm{H}, \mathrm{P}$ & $\mathrm{m}, \mathrm{f}, \mathrm{p}, \mathrm{l}$ & \\
\hline $\mathrm{L} 1 / 40-41$ & Goeldichironomus & maculatus & $\begin{array}{l}\text { Trivinho-Strixino and } \\
\text { Strixino, 1991b: } 593\end{array}$ & $\mathrm{H}, \mathrm{P}$ & $\mathrm{m}, \mathrm{f}$ & \\
\hline $\mathrm{L} 1 / 30-36$ & Goeldichironomus & neopictus & $\begin{array}{l}\text { Trivinho-Strixino and } \\
\text { Strixino, 1998: } 271\end{array}$ & $\mathrm{H}, \mathrm{P}$ & $\mathrm{m}, \mathrm{f}, \mathrm{p}, \mathrm{l}$ & \\
\hline $\mathrm{L} 1 / 14-20$ & Goeldichironomus & petiolicola & $\begin{array}{l}\text { Trivinho-Strixino and } \\
\text { Strixino, 2005: } 442\end{array}$ & $\mathrm{H}, \mathrm{P}$ & $\mathrm{m}, \mathrm{f}, \mathrm{p}, \mathrm{l}$ & \\
\hline $\mathrm{L} 2 / 01$ & Nimbocera & paulensis & $\begin{array}{l}\text { Trivinho-Strixino and } \\
\text { Strixino, 1991a: } 175\end{array}$ & $\mathrm{H}, \mathrm{P}$ & 1 & 3 \\
\hline M1/36 & Nimbocera & rhabdomantis & $\begin{array}{l}\text { Trivinho-Strixino and } \\
\text { Strixino, 1991a: } 173\end{array}$ & $\mathrm{H}, \mathrm{P}$ & 1 & 4 \\
\hline
\end{tabular}




\begin{tabular}{|c|c|c|c|c|c|}
\hline $\begin{array}{l}\text { Type } \\
\text { number }\end{array}$ & Original genus & Original species & $\begin{array}{l}\text { Author/ } \\
\text { Reference }\end{array}$ & Type & $\begin{array}{l}\text { Sex/Life Note } \\
\text { stage }\end{array}$ \\
\hline $\mathrm{L} 1 / 47-48$ & Oukuriella & jatai & $\begin{array}{l}\text { Trivinho-Strixino and } \\
\text { Messias 2005: } 285\end{array}$ & $\mathrm{H}, \mathrm{P}$ & $\mathrm{m}, \mathrm{f}, \mathrm{p}, \mathrm{l}$ \\
\hline A $1 / 25-32$ & Parachironomus & lupus & $\begin{array}{l}\text { Trivinho-Strixino, } \\
\text { Silva and Roque, } \\
\text { 2010: } 2\end{array}$ & $\mathrm{H}, \mathrm{P}$ & $\mathrm{m}, \mathrm{p}, 1$ \\
\hline A3/16-25 & Paratanytarsus & corbii & $\begin{array}{l}\text { Trivinho-Strixino, } \\
\text { 2010: } 60\end{array}$ & $\mathrm{H}, \mathrm{P}$ & $\mathrm{m}, \mathrm{f}, \mathrm{p}, \mathrm{l}$ \\
\hline A3/14-15 & Paratanytarsus & silentii & $\begin{array}{l}\text { Trivinho-Strixino, } \\
\text { 2010: } 65\end{array}$ & $\mathrm{H}, \mathrm{P}$ & $\mathrm{m}$ \\
\hline $\mathrm{A} 2 / 25-30$ & Pelomus & psammophilus & $\begin{array}{l}\text { Trivinho-Strixino and } \\
\text { Strixino, 2008: } 224\end{array}$ & $\mathrm{H}, \mathrm{P}$ & $\mathrm{m}, \mathrm{f}, \mathrm{p}, \mathrm{l}$ \\
\hline A2/01-05 & Pelomus & sophiae & $\begin{array}{l}\text { Trivinho-Strixino and } \\
\text { Silva 2011: } 274\end{array}$ & $\mathrm{H}, \mathrm{P}$ & $\mathrm{m}, \mathrm{p}, \mathrm{l}$ \\
\hline $\mathrm{O} 2 / 48-50$ & Riethia & manauara & $\begin{array}{l}\text { Neubern, Trivinho- } \\
\text { Strixino and Silva, } \\
\text { 2011: } 596\end{array}$ & $\mathrm{H}, \mathrm{P}$ & $\mathrm{m}, \mathrm{p}, 1$ \\
\hline $\mathrm{H} 1 / 23-25$ & Tanytarsus & alfredoi & $\begin{array}{l}\text { Sanseverino and } \\
\text { Trivinho-Strixino, } \\
\text { 2010: } 78\end{array}$ & $\mathrm{H}, \mathrm{P}$ & $\mathrm{m}, \mathrm{p}, 1$ \\
\hline $\mathrm{H} 2 / 01-20$ & Tanytarsus & caipira & $\begin{array}{l}\text { Trivinho-Strixino and } \\
\text { Strixino, 2007: } 62\end{array}$ & $\mathrm{H}, \mathrm{P}$ & $\mathrm{m}, \mathrm{f}, \mathrm{p}, \mathrm{l}$ \\
\hline $\mathrm{H} 2 / 43-48$ & Tanytarsus & corumba & $\begin{array}{l}\text { Trivinho-Strixino, } \\
\text { Wiedenbrug and Silva, } \\
\text { 2015: } 92\end{array}$ & $\mathrm{H}, \mathrm{P}$ & $\mathrm{m}$ \\
\hline $\mathrm{H} 1 / 29-30$ & Tanytarsus & fittkaui & $\begin{array}{l}\text { Sanseverino and } \\
\text { Trivinho-Strixino, } \\
\text { 2010: } 75\end{array}$ & $\mathrm{H}, \mathrm{P}$ & $\mathrm{m}, \mathrm{p}, \mathrm{l}$ \\
\hline $\mathrm{H} 1 / 33-43$ & Tanytarsus & giovannii & $\begin{array}{l}\text { Sanseverino and } \\
\text { Trivinho-Strixino, } \\
\text { 2010: } 71\end{array}$ & $\mathrm{H}, \mathrm{P}$ & $\mathrm{m}, \mathrm{p}, \mathrm{l}$ \\
\hline H3/33-37 & Tanytarsus & hirsutus & $\begin{array}{l}\text { Trivinho-Strixino, } \\
\text { Wiedenbrug and Silva, } \\
\text { 2015: } 93\end{array}$ & $\mathrm{H}, \mathrm{P}$ & $\mathrm{m}, \mathrm{p}$ \\
\hline $\mathrm{H} 3 / 01-13$ & Tanytarsus & impar & $\begin{array}{l}\text { Trivinho-Strixino and } \\
\text { Strixino, 2004: } 160\end{array}$ & $\mathrm{H}, \mathrm{P}$ & $\mathrm{m}, \mathrm{f}, \mathrm{p}, \mathrm{l}$ \\
\hline $\mathrm{H} 3 / 40-43$ & Tanytarsus & jatai & $\begin{array}{l}\text { Trivinho-Strixino, } \\
\text { Wiedenbrug and Silva, } \\
\text { 2015: } 95\end{array}$ & $\mathrm{H}, \mathrm{P}$ & $\mathrm{m}$ \\
\hline H1/46-49 & Tanytarsus & lenyae & $\begin{array}{l}\text { Sanseverino and } \\
\text { Trivinho-Strixino, } \\
\text { 2010: } 68\end{array}$ & $\mathrm{H}, \mathrm{P}$ & $\mathrm{m}, \mathrm{p}$ \\
\hline $\mathrm{H} 2 / 29-36$ & Tanytarsus & longitubuli & $\begin{array}{l}\text { Trivinho-Strixino, } \\
\text { Wiedenbrug and Silva, } \\
\text { 2015: } 96\end{array}$ & $\mathrm{H}, \mathrm{P}$ & $\mathrm{m}, \mathrm{p}, \mathrm{l}$ \\
\hline H3/19-27 & Tanytarsus & magnus & $\begin{array}{l}\text { Trivinho-Strixino and } \\
\text { Strixino, 2004: } 155\end{array}$ & $\mathrm{H}, \mathrm{P}$ & $\mathrm{m}, \mathrm{f}, \mathrm{p}, \mathrm{l}$ \\
\hline $\mathrm{H} 1 / 01-21$ & Tanytarsus & obiriciae & $\begin{array}{l}\text { Trivinho-Strixino and } \\
\text { Sonoda 2006: } 2\end{array}$ & $\mathrm{H}, \mathrm{P}$ & $\mathrm{m}, \mathrm{f}, \mathrm{p}, \mathrm{l}$ \\
\hline
\end{tabular}




\begin{tabular}{|c|c|c|c|c|c|c|}
\hline $\begin{array}{l}\text { Type } \\
\text { number }\end{array}$ & Original genus & Original species & $\begin{array}{l}\text { Author/ } \\
\text { Reference }\end{array}$ & Type & $\begin{array}{l}\text { Sex/Life } \\
\text { stage }\end{array}$ & Note \\
\hline $\mathrm{H} 3 / 30-31$ & Tanytarsus & pseudocurvicristatus & $\begin{array}{l}\text { Trivinho-Strixino, } \\
\text { Wiedenbrug and Silva, } \\
\text { 2015: } 98\end{array}$ & $\mathrm{H}, \mathrm{P}$ & $\mathrm{m}, \mathrm{p}, 1$ & \\
\hline A3/01-05 & Tapajos & cristinae & $\begin{array}{l}\text { Trivinho-Strixino, } \\
\text { Silva and Oliveira, } \\
\text { 2013: } 396\end{array}$ & $\mathrm{H}, \mathrm{P}$ & $\mathrm{m}$ & \\
\hline M3/01-15 & Xenochironomus & ceciliae & $\begin{array}{l}\text { Roque and Trivinho- } \\
\text { Strixino, 2005: } 232\end{array}$ & $\mathrm{H}, \mathrm{P}$ & $\mathrm{m}, \mathrm{f}, \mathrm{p}, \mathrm{l}$ & \\
\hline
\end{tabular}

\section{Orthocladiinae}

\begin{tabular}{|c|c|c|c|c|c|}
\hline $\mathrm{N} 3 / 01$ & Gravatamberus & nidularium & $\begin{array}{l}\text { Mendes and Andersen, } \\
\text { 2008: } 49\end{array}$ & $\mathrm{P}$ & $\mathrm{m}, \mathrm{p}, \mathrm{l}$ \\
\hline $\mathrm{N} 3 / 02$ & Physoneura & paulseni & $\begin{array}{l}\text { Stur and Andersen, } \\
\text { 2000: } 132\end{array}$ & $\mathrm{P}$ & $\mathrm{m}$ \\
\hline
\end{tabular}

Podonominae

\begin{tabular}{|c|c|c|c|c|c|}
\hline $\mathrm{N} 1 / 13-14$ & Podonomus & pepinellii & $\begin{array}{l}\text { Roque and Trivinho- } \\
\text { Strixino, 2004: } 2\end{array}$ & $\mathrm{H}$ & $\mathrm{p}$ \\
\hline \multicolumn{6}{|c|}{ Tanypodinae } \\
\hline $\mathrm{P} 1 / 25$ & Ablabesmyia & arquata & Neubern, 2013: 9 & $\mathrm{H}$ & $\mathrm{m}$ \\
\hline P3/02-06 & Ablabesmyia & cauame & Neubern, 2013: 13 & $\mathrm{H}, \mathrm{P}$ & $\mathrm{m}$ \\
\hline $\mathrm{P} 1 / 12-20$ & Ablabesmyia & commata & Neubern, 2013: 21 & $\mathrm{H}, \mathrm{P}$ & $\mathrm{m}$ \\
\hline O1/01-21 & Ablabesmyia & communiba & Neubern, 2013: 24 & $\mathrm{H}, \mathrm{P}$ & $\mathrm{m}, \mathrm{p}, \mathrm{l}$ \\
\hline P2/12-14 & Ablabesmyia & cordeiroi & Neubern, 2013: 30 & $\mathrm{H}, \mathrm{P}$ & $\mathrm{m}$ \\
\hline $\mathrm{O} 2 / 37-38$ & Ablabesmyia & depaulai & Neubern, 2013: 33 & $\mathrm{H}$ & $\mathrm{m}, \mathrm{p}, 1$ \\
\hline P2/40-42 & Ablabesmyia & diversa & Neubern, 2013: 39 & $\mathrm{H}, \mathrm{P}$ & $\mathrm{m}$ \\
\hline $\mathrm{O} 2 / 01-05$ & Ablabesmyia & ducke & Neubern, 2013: 41 & $\mathrm{H}, \mathrm{P}$ & $\mathrm{m}, \mathrm{p}, \mathrm{l}$ \\
\hline $\mathrm{O} 2 / 32-33$ & Ablabesmyia & fazzari & Neubern, 2013: 47 & $\mathrm{H}, \mathrm{P}$ & $\mathrm{m}, \mathrm{p}, 1$ \\
\hline $\mathrm{P} 1 / 45-46$ & Ablabesmyia & fusariae & Neubern, 2013: 53 & $\mathrm{H}, \mathrm{P}$ & $\mathrm{m}, \mathrm{p}, \mathrm{l}$ \\
\hline $\mathrm{P} 1 / 36-39$ & Ablabesmyia & gessnerae & Neubern, 2013: 59 & $\mathrm{H}, \mathrm{P}$ & $\mathrm{m}, \mathrm{p}, \mathrm{l}$ \\
\hline$P 2 / 33-37$ & Ablabesmyia & gigas & Neubern, 2013: 64 & $\mathrm{H}, \mathrm{P}$ & $\mathrm{m}$ \\
\hline $\mathrm{O} 2 / 29$ & Ablabesmyia & jaquirana & Neubern, 2013: 71 & $\mathrm{H}$ & $\mathrm{m}$ \\
\hline
\end{tabular}




\begin{tabular}{|c|c|c|c|c|c|c|}
\hline $\begin{array}{l}\text { Type } \\
\text { number }\end{array}$ & Original genus & Original species & $\begin{array}{l}\text { Author/ } \\
\text { Reference }\end{array}$ & Type & $\begin{array}{l}\text { Sex/Life } \\
\text { stage }\end{array}$ & Note \\
\hline $\mathrm{P} 2 / 18$ & Ablabesmyia & manauara & Neubern, 2013: 80 & $\mathrm{H}$ & $\mathrm{m}$ & \\
\hline $\mathrm{P} 2 / 20-22$ & Ablabesmyia & martha & Neubern, 2013: 83 & $\mathrm{H}, \mathrm{P}$ & $\mathrm{m}$ & \\
\hline P3/09-10 & Ablabesmyia & novema & Neubern, 2013: 89 & $\mathrm{H}, \mathrm{P}$ & $\mathrm{m}, \mathrm{p}, 1$ & \\
\hline $\mathrm{O} 3 / 12-16$ & Ablabesmyia & oliveirai & $\begin{array}{l}\text { Oliveira and Fonseca- } \\
\text { Gessner, 2006: } 740\end{array}$ & $\mathrm{H}, \mathrm{P}$ & $\mathrm{m}, \mathrm{p}, 1$ & \\
\hline P1/29-33 & Ablabesmyia & parannulata & Neubern, 2013: 95 & $\mathrm{H}, \mathrm{P}$ & $\mathrm{m}$ & \\
\hline $\mathrm{O} 2 / 39-40$ & Ablabesmyia & parareissi & Neubern, 2013: 99 & $\mathrm{H}, \mathrm{P}$ & $\mathrm{m}$ & \\
\hline $\mathrm{O} 2 / 20-27$ & Ablabesmyia & pinhoi & Neubern, 2013: 102 & $\mathrm{H}, \mathrm{P}$ & $\mathrm{m}, \mathrm{p}$ & \\
\hline $\mathrm{P} 2 / 30$ & Ablabesmyia & rafaeli & Neubern, 2013: 106 & $\mathrm{H}$ & $\mathrm{m}$ & \\
\hline $\mathrm{P} 1 / 42$ & Ablabesmyia & separata & Neubern, 2013: 109 & $\mathrm{H}$ & $\mathrm{m}$ & \\
\hline $\mathrm{P} 2 / 25-27$ & Ablabesmyia & strixinoae & Neubern, 2013: 113 & $\mathrm{H}, \mathrm{P}$ & $\mathrm{m}, \mathrm{p}, 1$ & \\
\hline $\mathrm{P} 1 / 21-23$ & Ablabesmyia & suiamissu & Neubern, 2013: 118 & $\mathrm{H}, \mathrm{P}$ & $\mathrm{m}$ & \\
\hline O1/41-42 & Clinotanypus & caritus & $\begin{array}{l}\text { Oliveira, Silva and } \\
\text { Trivinho-Strixino, } \\
\text { 2014a: } 319\end{array}$ & $\mathrm{P}$ & $\mathrm{m}, \mathrm{p}, 1$ & \\
\hline O1/31-32 & Clinotanypus & gymnos & $\begin{array}{l}\text { Oliveira, Silva and } \\
\text { Trivinho-Strixino, } \\
\text { 2014a: } 325\end{array}$ & $\mathrm{H}, \mathrm{P}$ & $\mathrm{m}, \mathrm{p}, 1$ & \\
\hline O1/44-45 & Clinotanypus & setosus & $\begin{array}{l}\text { Oliveira, Silva and } \\
\text { Trivinho-Strixino, } \\
\text { 2014a: } 330\end{array}$ & $\mathrm{H}, \mathrm{P}$ & $\mathrm{m}, \mathrm{p}, \mathrm{l}$ & \\
\hline O1/34-39 & Clinotanypus & striatus & $\begin{array}{l}\text { Oliveira, Silva and } \\
\text { Trivinho-Strixino, } \\
\text { 2014a: } 336\end{array}$ & $\mathrm{H}, \mathrm{P}$ & $\mathrm{m}, \mathrm{p}, \mathrm{l}$ & \\
\hline B3/11-14 & Guassutanypus & oliveirai & $\begin{array}{l}\text { Roque and Trivinho- } \\
\text { Strixino, 2003: } 161\end{array}$ & $\mathrm{H}, \mathrm{P}$ & $\mathrm{m}, \mathrm{f}, \mathrm{p}, \mathrm{l}$ & 5 \\
\hline $\mathrm{O} 3 / 04-10$ & Hudsonimyia & $\operatorname{araxa}$ & $\begin{array}{l}\text { Silva, Wiedenbrug, } \\
\text { Oliveira, Trivinho- } \\
\text { Strixino and Pepinelli, } \\
\text { 2012: } 1629\end{array}$ & $\mathrm{P}$ & $\mathrm{m}, \mathrm{f}, \mathrm{p}, \mathrm{l}$ & \\
\hline $\mathrm{O} 3 / 01$ & Hudsonimyia & caissara & $\begin{array}{l}\text { Silva, Wiedenbrug, } \\
\text { Oliveira, Trivinho- } \\
\text { Strixino and Pepinelli, } \\
\text { 2012: } 1623\end{array}$ & $\mathrm{P}$ & $\mathrm{m}, \mathrm{p}, \mathrm{l}$ & \\
\hline $\mathrm{O} 2 / 42-44$ & Larsia & gelhausi & $\begin{array}{l}\text { Oliveira and Silva, } \\
\text { 2011: } 30\end{array}$ & $\mathrm{H}, \mathrm{P}$ & $\mathrm{m}, \mathrm{p}, 1$ & \\
\hline
\end{tabular}




\begin{tabular}{|c|c|c|c|c|c|c|}
\hline $\begin{array}{l}\text { Type } \\
\text { number }\end{array}$ & Original genus & Original species & $\begin{array}{l}\text { Author/ } \\
\text { Reference }\end{array}$ & Type & $\begin{array}{l}\text { Sex/Life } \\
\text { stage }\end{array}$ & Note \\
\hline $\mathrm{O} 2 / 12-14$ & Larsia & hamadae & $\begin{array}{l}\text { Oliveira and Silva, } \\
\text { 2011: } 35\end{array}$ & $\mathrm{H}$ & $\mathrm{m}, \mathrm{p}, \mathrm{l}$ & \\
\hline B3/07-08 & Larsia & labartheae & $\begin{array}{l}\text { Serpa-Filho, 2005: } \\
295\end{array}$ & $P$ & $\mathrm{~m}$ & \\
\hline $\mathrm{O} 3 / 25-32$ & Metapelopia & corbii & $\begin{array}{l}\text { Silva, Oliveira and } \\
\text { Trivinho-Strixino, } \\
\text { 2014: } 86\end{array}$ & $\mathrm{H}, \mathrm{P}$ & $\mathrm{m}, \mathrm{f}, \mathrm{p}, \mathrm{l}$ & \\
\hline $\mathrm{O} 2 / 07-10$ & Monopelopia & paranaensis & $\begin{array}{l}\text { Oliveira, Mendes and } \\
\text { Silva, 2010: } 54\end{array}$ & $\mathrm{H}, \mathrm{P}$ & $\mathrm{m}, \mathrm{f}, \mathrm{p}, \mathrm{l}$ & \\
\hline O1/24-27 & Parapentaneura & brunnescens & $\begin{array}{l}\text { Oliveira, Silva and } \\
\text { Trivinho-Strixino, } \\
\text { 2014b: } 27\end{array}$ & $\mathrm{H}, \mathrm{P}$ & $\mathrm{m}, \mathrm{p}, \mathrm{l}$ & \\
\hline O1/29 & Parapentaneura & flavescens & $\begin{array}{l}\text { Oliveira, Silva and } \\
\text { Trivinho-Strixino, } \\
\text { 2014b: } 31\end{array}$ & $\mathrm{H}$ & $\mathrm{m}, \mathrm{p}, \mathrm{l}$ & \\
\hline
\end{tabular}

Telmatogetoninae

\begin{tabular}{lll}
\hline & & Oliveira, Silva and \\
P1/01-09 Thalassomya & Trivinho-Strixino, H, P m, p, 1 \\
& $2013: 590$ \\
\hline
\end{tabular}

Notes in Table 1:

1. In Chironomus amissum Correia, TrivinhoStrixino and Michailova, 2013, the original spelling of the species epithet, "amissum", meaning loss, has to be emended. Correia et al. (2012: 134) gave an etymological derivation from a Latin noun "amissum", meaning loss, but that Latin noun is "amissus", with masculine rather than neuter gender and ending.

2. Chironomus sancticaroli Trivinho-Strixino and Strixino, 1981 has been treated as a junior synonym of C. xanthus Rempel, 1939 by some authors since Spies and Reiss (1996).

3. Nimbocera paulensis Trivinho-Strixino and Strixino, 1991 has been treated as a junior synonym of Caladomyia ortoni Säwedal, 1981 since Trivinho-Strixino and Strixino (2003).

4. Nimbocera rhabdomantis Trivinho-Strixino and Strixino, 1991 has been carried as Tanytarsus rhabdomantis (Trivinho-Strixino and Strixino, 1991) since Trivinho-Strixino and Sanseverino (2003).

5. Guassutanypus oliveirai Roque and TrivinhoStrixino, 2003 has been carried as Alotanypus oliveirai (Roque and Trivinho-Strixino, 2003) since Cranston and Epler (2013).

\section{Acknowledgments}

We are grateful to two reviewers for the thorough reading and constructive comments to our manuscript. The visit of the present first author to the Laboratory of Ecology of Aquatic Insects (LEIA - UFSCar) was possible thanks to the generosity of George Putnam, through a Putnam expedition Grant from the Museum of Comparative Zoology at Harvard University. F. L. Silva was also supported by a Postdoctoral Fellowship from the Coordination for the Improvement of Higher Education Personnel (CAPES).

\section{References}

Correia, L.S.C. and Trivinho-Strixino, S. 2007. New species of Chironomus Meigen (Diptera: Chironomidae: Chironominae) from Brazil. Zootaxa 1504: 53-68.

Correia, L.C.S., Trivinho-Strixino, S. and Michailova P. 2005. A new species of Chironomus Meigen, 1803 (Diptera, Chironomidae) from the southeast of Brazil. - Studies on Neotropical Fauna and Environment 40(1): 29-38.

Correia, L.C.S., Trivinho-Strixino, S. and Michailova, P. 2006. A new species of Chironomus Meigen (Diptera: Chironomidae: Chironominae) from polluted streams of southeastern Brazil. - Zootaxa 1130: 57-68. 
Correia, L., Trivinho-Strixino, S. and Michailova, P. 2013. Chironomus amissum sp. n. (Diptera, Chironomidae) from southeastern Brazil. - $B i$ ota Neotropica 13(4): 133-138.

Cranston, P.S. and Epler, J.H. 2013. The larvae of Tanypodinae (Diptera: Chironomidae) of the Holarctic region - Keys and diagnoses. In Andersen, T., Cranston, P.S. and Epler, J.H. (Eds) Chironomidae of the Holarctic Region - Keys and diagnoses. Part 1. Larvae. - Insect Systematics \& Evolution, Supplement 66: 39-136.

Mendes, H.F. and Andersen, T. 2008. A review of Antillocladius Sæther and Litocladius Mendes, Andersen et Sæther, with the description of two new Neotropical genera (Diptera, Chironomidae, Orthocladiinae). - Zootaxa 1887: 1-75.

Neubern, C.S.O., Navarro M.A.S. and FonsecaGessner A.A. 2013. Neotropical Ablabesmyia Johannsen (Diptera: Chironomidae, Tanypodinae) - Part I. - Zootaxa 3733: 1-123.

Neubern, C.S.O, Trivinho-Strixino, S. and Silva, F.L. 2011. Riethia manauara n. sp., an Amazonian chironomid (Diptera: Chironomidae) from Brazil. - Neotropical Entomology 40(5): 595-599.

Oliveira, C.S.N. and Fonseca-Gessner, A.A. 2006. New species of Ablabesmyia Johannsen, 1905 (Diptera, Chironomidae, Tanypodinae) from the Neotropical region, with description of male adults and immature stages. - Revista Brasileira de Zoologia 23: 740-745.

Oliveira, C.S.N. and Silva, F.L. 2011. Two new species of Larsia Fittkau, 1962 (Diptera: Chironomidae: Tanypodinae) from Neotropical Region, with a checklist of Larsia species of the world. - Zootaxa 2786: 27-41.

Oliveira, C.S.N, Mendes, H.F. and Navarro, M.A.S. 2010. Monopelopia paranaense, a new tanypod species from South, Brazil, with keys to the Neotropical - Nearctic species (Diptera: Chironomidae). - Zootaxa 2420: 53-62.

Oliveira, C.S.N., Silva, F.L. and Trivinho-Strixino, S. 2013. Thalassomya gutae sp. n., a new marine chironomid (Diptera: Chironomidae: Telmatogetoninae) from the Brazilian coast. Zootaxa 3701(5): 589-595.

Oliveira, C.S.N., Silva, F.L. and Trivinho-Strixino, S. 2014a. Four new species of Clinotanypus Kieffer, 1913 (Diptera: Chironomidae: Tanypodinae) from Neotropical region. - Journal of Natural History 48: 317-343.
Oliveira, C.S.N., Silva, F.L. and Trivinho-Strixino, S. 2014b. Two new species of Parapentaneura (Diptera: Chironomidae: Tanypodinae) from Brazil, with keys to the males and immature stages. - Studies on Neotropical Fauna and Environment 49: 26-35.

Pape, T. and Thompson, F.C. (Eds) 2013. Systema Dipterorum, version 1.5. http://www.diptera. org/ [last accessed on 14/08/2015]

Roque, F.O. and Trivinho-Strixino, S. 2003. Guassutanypus oliveirai, a new genus and species of Macropelopiini from Brazil (Insecta, Diptera, Chironomidae). - Spixiana,26(2): 159-164.

Roque, F.O. and Trivinho-Strixino, S. 2004. Podonomus pepinellii $\mathrm{n}$. sp., first record of the genus and subfamily from Brazil (Diptera: Chironomidae: Podonominae). - Zootaxa 689: 1-7.

Roque, F.O. and Trivinho-Strixino, S. 2005. Xenochironomus ceciliae (Diptera: Chironomidae), a new chironomid species inhabiting freshwater sponges in Brazil. - Hydrobiologia 534: 231-238.

Roque, F.O. and Trivinho-Strixino, S. 2008. Four new species of Endotribelos Grodhaus, a common fallen fruit-dwelling chironomid genus in Brazilian streams (Diptera: Chironomidae; Chironomidae). - Studies on Neotropical Fauna and Environment 43(3): 191-207.

Sanseverino, A.M. and Trivinho-Strixino, S. 2010. New species of Tanytarsus van der Wulp (Diptera: Chironomidae) from São Paulo State, Brazil. - Neotropical Entomology 39(1): 67-82.

Sanseverino, A.M., Trivinho-Strixino, S. and Nessimian, J.L. 2010. Taxonomic status of Nimbocera Reiss, 1972, a junior synonym of Tanytarsus van der Wulp, 1874 (Diptera: Chironomidae). - Zootaxa, 2359: 43-57.

Serpa-Filho, A. 2005. Sobre uma nova espécie neotrópica do gênero Larsia Fittkau, 1962 (Diptera: Chironomidae: Tanypodinae). - Entomología y Vectores 12(2): 293-302.

Silva, F.L., Oliveira, C.S.N. and Trivinho-Strixino, S. 2014. Metapelopia corbii gen. n., sp. n., a new Tanypodinae (Diptera: Chironomidae) from the Neotropical Region. - Annales de Limnologie - International Journal of Limnology 50: 85-95.

Silva, F.L., Trivinho-Strixino, S. and Oliveira, H.R.N. 2010. New species of Cryptochironomus Kieffer, 1918 (Diptera: Chironomidae: Chironominae) from Brazil. - Zootaxa 2614: 18-32. 
Silva, F.L., Wiedenbrug, S., Trivinho-Strixino, S., Oliveira C.S.N. Pepinelli M. 2012. Two new species of Hudsonimyia Roback, 1979 (Diptera: Chironomidae: Tanypodinae) from Neotropical Region unveiled by morphology and DNA barcoding. - Journal of Natural History 46: $1615-1638$

Spies, M. and Reiss, F. 1996. Catalog and bibliography of Neotropical and Mexican Chironomidae (Insecta, Diptera). - Spixiana, Supplement 22: 61-119.

Stur, E. and Andersen, T. 2000. A new species of Physoneura Ferrington and Sæther, 1995, from Ecuador (Chironomidae, Orthocladiinae). Norwegian Journal of Entomology 47: 131136.

Trivinho-Strixino, S. 1997. Nova espécie do gênero Aedokritus, com descrição das formas imaturas. - Revista Brasileira de Entomologia 41(1): 13-16.

Trivinho-Strixino, S. 2010. Two new species of Paratanytarsus (Diptera: Chironomidae) from southeast of Brazil. - Zootaxa 2726: 59-67

Trivinho-Strixino S. 2012. A systematic review of Neotropical Caladomyia Säwedal (Diptera: Chironomidae). - Zootaxa 3495: 1-41.

Trivinho-Strixino, S. and Messias, M.C. 2005. A new species of Oukuriella Epler, 1986 (Insecta, Diptera, Chironomidae, Chironominae) from São Paulo state, Brazil. - Entomologia y Vectores 12(2): 283-291.

Trivinho-Strixino, S. and Pepinelli, M. 2015. A systematic study on Endotribelos Grodhaus (Diptera: Chironomidae) from Brazil including DNA barcoding to link males and females. Zootaxa 3936(1): 1-41.

Trivinho-Strixino, S. and Sanseverino, A.M. 2003. Tanytarsus rhabdomantis: new combination for Nimbocera rhabdomantis TrivinhoStrixino and Strixino, 1991 (Diptera: Chironomidae). - Zootaxa 389: 1-10.

Trivinho-Strixino, S. and Silva, F.L. 2011. A new species of Pelomus Reiss, 1989 (Diptera: Chironomidae: Chironominae) from the Neotropical Region, with emendation of the generic diagnosis. - Aquatic Insects 33(3): 273-279.

Trivinho-Strixino, S. and Siqueira, T. 2007. New species of Beardius Reiss et Sublette, 1985 (Diptera: Chironomidae) from Southeastern Brazil. In: Andersen, T. (Ed.) Contributions to the Systematics and Ecology of Aquatic Dip- tera - A Tribute to Ole A. Sæther, The Caddis Press, pp. 281-286.

Trivinho-Strixino, S. and Sonoda, K.C. 2006. A new Tanytarsus species (Insecta, Diptera, Chironomidae) from São Paulo State, Brazil. - Biota Neotropica 6(2): 1-9.

Trivinho-Strixino, S. and Strixino, G. 1981. Nova espécie do gênero Chironomus Meigen do Sul do Brasil (Diptera: Chironomidae). - Revista Brasileira de Entomologia 25(4): 333-340.

Trivinho-Strixino, S. and Strixino, G. 1991a. Duas novas espécies de Nimbocera Reiss (Diptera, Chironomidae) do Estado de São Paulo, Brasil. - Revista Brasileira de Entomologia 35(1): 173-178.

Trivinho-Strixino, S. and Strixino, G. 1991b. Nova espécie de Goeldichironomus Fittkau (Diptera, Chironomidae) do Brasil. - Revista Brasileira de Entomologia 35(3): 593-602.

Trivinho-Strixino, S. and Strixino, G. 1998. Goeldichironomus neopictus, a new species from the southeast of Brazil: description and bionomic information (Insecta, Diptera, Chironomidae). - Spixiana 21(3): 271-278.

Trivinho-Strixino, S. and Strixino, G. 2000a. A new species of Caladomyia Säwedal, 1981, with description of the female and immature stages (Insecta, Diptera, Chironomidae). Spixiana 23(2): 167-173.

Trivinho-Strixino, S. and Strixino, G. 2000b. Two new species of Beardius Reiss et Sublette (Diptera, Chironomidae) from Southeastern Brazil. In: Late 20th Century Research on Chironomidae: an Anthology from the 13th International Symposium on Chironomidae, Shaker Verlag, pp. 245-250.

Trivinho-Strixino, S. and Strixino, G. 2003. The immature stages of two Caladomyia Säwedal, 1981 species, from São Paulo State, Brazil (Chironomidae, Chironominae, Tanytarsini). Revista Brasileira de Entomologia 47(4): 597602.

Trivinho-Strixino, S. and Strixino, G. 2004. Two new species of Tanytarsus from Southeast of Brazil (Insecta, Diptera, Chironomidae). Spixiana 27(2): 155-164.

Trivinho-Strixino, S. and Strixino, G. 2005. Two new species of Goeldichironomus Fittkau from southeast Brazil (Diptera, Chironomidae). Revista Brasileira de Entomologia 49(4): 441445. 
Trivinho-Strixino, S. and Strixino, G. 2007. A new Neotropical species of Tanytarsus van der Wulp, 1874 (Diptera: Chironomidae) with an unusual anal process. - Zootaxa 1654: 61-67.

Trivinho-Strixino, S. and Strixino, G. 2008. A new species of Pelomus Reiss, 1989 (Diptera: Chironomidae) from southeastern Brazil, with the description of immature stages. - Boletim do Museu Municipal de Funchal, Suplemento 13: 223-231.

Trivinho-Strixino, S., Silva, F.L and Oliveira, C.S.N. 2013. Tapajos cristinae n. gen., n. sp. (Diptera: Chironomidae: Chironominae) from the Neotropical Region. - Zootaxa 3710(4): 395-399.
Trivinho-Strixino, S., Silva, F.L. and Roque, F.O. 2010. A new species of Parachironomus Lenz, 1921 (Diptera: Chironomidae: Chironominae), and description of immature stages of two other species from the Neotropical Region. Zootaxa 2689: 1-14.

Trivinho-Strixino, S., Wiedenbrug, S. and Silva, F.L. 2015. New species of Tanytarsus van der Wulp (Diptera: Chironomidae: Tanytarsini) from Brazil. - European Journal of Environmental Sciences,5(1): 92-100.

Article submitted 18. September 2015, accepted 27. October 2015, published 22. December 2015. 